\title{
Quantum Theory of Gravitation and Locality Postulate*
}

\author{
K. Kraus \\ Institut für Theoretische Physik der Universität Marburg
}

Received January 29, 1968

\begin{abstract}
A quantized space-time metric $g_{i k}(x)$ is investigated within a suitably modified axiomatic approach. Coordinate distances $d x$ are called absolutely spacelike if $g_{i k}(x) d x^{i} d x^{k}$ is negative definite. For such distances, fields are assumed to commute or anticommute, respectively (generalized locality). The quantum fluctuations of the light cone $g_{i k}(x) d x^{i} d x^{k}=0$ are shown to extend to distances $d x$ which are space-like with respect to the Minkowski metric. Generalized locality is therefore weaker than the usual locality postulate.
\end{abstract}

\section{Quantized Gravity and Wightman Axioms}

A heuristic attempt is presented to describe a quantized space-time metric (i.e., quantized gravitational potentials) $g_{i k}(x)$ according to general ideas of axiomatic field theory. For this purpose, a considerably weakened form of the usual field theory axiomatics (compare, e.g., Streater and Wightman [1]) is appropriate. A closely related approach to the quantum theory of gravitation has been proposed independently by STROCCHI [2]. The assumptions used here will be formulated and discussed with respect to their applicability to quantized gravity. Most problematic in this respect is the usual locality postulate, which is therefore treated separately in the next section.

Postulate 1 (Quantum Theory, Invariance, Spectrum). Pure states of the system are unit vectors $\Psi$ in a Hilbert space $\mathscr{H}$ with positive norm. The inhomogeneous Lorentz group (or, more precisely, its covering group) is continuously represented in $\mathscr{H}$ by unitary operators $U(a, \Lambda)$. With $U(a, 1)=e^{i P_{k} a^{k}}$, the spectrum of $P_{k}$ is contained in the closed forward light cone. A vacuum state $\Psi_{0}$, defined by $U(a, \Lambda) \Psi_{0}=\Psi_{0}$, exists and is unique. -

This postulate becomes meaningful only with a suitable interpretation of $U(a, \Lambda)$, since a representation $U(a, \Lambda)$ with the required properties can be constructed formally in any infinite-dimensional Hilbert space $\mathscr{H}$.

* Supported in part by the Deutsche Forschungsgemeinschaft. 
Under suitable asymptotic conditions, the theory considered will describe scattering situations, with incoming and outgoing free particles including gravitons. As usual, this leads to representations $U_{\text {in }}(a, \Lambda)$ and $U_{\text {out }}(a, \Lambda)$, with an obvious physical interpretation, in the Fock spaces $\mathscr{H}_{\text {in }}$ and $\mathscr{H}_{\text {out }}$ of scattering states. Completeness of the scattering states, $\mathscr{H}_{\text {in }}=\mathscr{H}_{\text {out }}=\mathscr{H}$, and Lorentz invariance of the $S$-matrix, $U_{\text {in }}(a, \Lambda)=U_{\text {out }}(a, \Lambda)=U(a, \Lambda)$, then immediately lead to Postulate 1.

This argument also explains the role of Lorentz invariance in a quantum theory of gravity. Asymptotic completeness, together with Lorentz invariant description of free gravitons (i.e., as mass zero, spin two particles) and Lorentz invariance of scattering amplitudes, are sufficient for Lorentz invariance of the whole theory. General covariance, on the other hand, can be understood as a kind of "gauge" invariance of the classical theory, similar to gauge invariance in electrodynamics. By analogy with the latter one should expect some "breaking" of gauge invariance if the theory is quantized, different gauges corresponding in general to different "quantizations" with different Hilbert spaces. Also in a quantum theory of gravity one should therefore expect neither less nor much more than Lorentz invariance.

With the asymptotic free particles one can associate incoming and outgoing free fields. The following postulate requires the existence of "interpolating" fields, which describe the dynamics of the system at finite times and, by asymptotic conditions, determine the $S$-matrix.

Postulate 2 (Fields, Cyclic Vacuum). Fields $\varphi_{\alpha}(x), \alpha=1 \ldots N$ are given, with components $\varphi_{\alpha K}(x), K=1 \ldots n_{\alpha}$ being strictly localizable operator-valued distributions in $\mathscr{H}$. The smeared fields $\varphi_{\alpha K}(f)$ $=\int f(x) \varphi_{\alpha K}(x) d^{4} x$ with arbitrary test functions $f(x)$, as well as arbitrary polynomials of such operators and their adjoints, can be applied to the vacuum state $\Psi_{0}$, thereby generating a domain $D$ which is dense in $\mathscr{H}$. -

More specifically, we will assume the existence of a "quantized spacetime metric"

$$
g_{i k}(x)=g_{k i}(x)=g_{i k}^{*}(x), \quad i, k=1 \ldots 4
$$

among the fields $\varphi_{\alpha}(x)$.

The field components $\varphi_{\alpha K}(x)$ are not assumed to be tempered or Schwartz distributions. Instead, only strict localizability as defined by $\mathrm{J}_{\mathrm{AFFE}}[3]$ is required. This could indeed be a necessary generalization if gravitational interactions would turn out to be non-renormalizable. However, some more specific assumptions about the test function space 
will be used in the following. The existence of non-negative test functions is required in Section II for the physical interpretation of $g_{i k}(x)$. A somewhat stronger assumption of this type will be used in Section III.

Obviously $D$ becomes a dense invariant domain of definition common to all operators $\varphi_{\alpha K}(f)$ and $\varphi_{\alpha K}^{*}(f)$. Little can be said to justify this assumption physically, since it is mainly a technical one, invented to permit application of WIGHTMAN's formalism [4].

The following postulate specifies the transformation law of the fields $\varphi_{\alpha}(x)$ with respect to space-time translations $U(a, 1)$. Usually one also fixes the transformation law with respect to Lorentz rotations $U(0, \Lambda)$. This, however, would here be very dangerous for the following reason.

In gauge invariant relativistic theories like electrodynamics and gravitation theory, two classes of "gauges" can be distinguished. Covariant gauges employ gauge conditions which are manifestly covariant with respect to Lorentz transformations. Examples of this are the Lorentz gauge of electrodynamics, and Fock's [5] harmonicity condition for gravity. Gauge conditions which are not manifestly Lorentz invariant define non-covariant gauges like, e.g., the Coulomb (radiation) gauge, or corresponding gauges of gravitation discussed by ARNowITT, DESER and Misner [6]. Only in covariant gauges the electromagnetic or gravitational potentials transform as a Lorentz vector or tensor, respectively.

Experience from quantum electrodynamics, as well as attempts to quantize gravity in Fock's gauge [7], seem to indicate an incompatibility of covariant gauge with asymptotic completeness (as shown by the occurrence of "unphysical particles"), or even with positive Hilbert space norm. In the framework of axiomatic field theory, this question has been analyzed recently by STROcCHI for electrodynamics [8] and for the linearized theory of gravitation [2]. Covariant gauges seem to require a reinterpretation, or even a reformulation, of Postulate 1. (Compare also WEINBERG's approach [9], where asymptotic completeness excludes manifest Lorentz covariance of the potentials corresponding to free photons and gravitons.)

We will therefore not assume a covariant gauge, but require simple transformation laws with respect to translations only.

Postulate 3 (Manifest Translation Covariance). The fields $\varphi_{\alpha}(x)$ transform under $U(a, 1)$ as

$$
U(a, 1) \varphi_{\alpha K}(x) U^{-1}(a, 1)=\varphi_{\alpha K}(x+a) .-
$$

Eq. (2) should be formulated, more precisely, in terms of smeared fields $\varphi_{\alpha K}(f)$, and is then required to hold on the domain $D$. By this postulate, $D$ becomes translation invariant, i.e., $U(a, 1) D=D$. 
By translation invariance, the $n$-point Wightman functions [1] are distributions in the $n-1$ coordinate differences only. Smearing with $n-1$ test functions is then sufficient to yield well-behaved functions of the remaining single coordinate. Therefore the expectation values $\left(\Psi, \varphi_{\alpha K}(x) \Psi\right)$ exist for all states $\Psi \in D$. In particular, any such state yields a well-behaved $c$-number metric

$$
g_{i k}(x \mid \Psi)=\left(\Psi, g_{i k}(x) \Psi\right) .
$$

By translation invariance of $\Psi_{0}$, the (mean) vacuum metric $g_{i k}\left(x \mid \Psi_{0}\right)$ is constant. Lorentz invariance and asymptotic condition should then suffice to yield

$$
g_{i k}\left(x \mid \Psi_{0}\right)=\eta_{i k}
$$

(Minkowski metric), even for non-covariant gauge. Eq. (4) holds trivially, of course, for covariant gauge.

Let us, moreover, assume all $c$-number metrics $g_{i k}(x \mid \Psi)$ to possess the same signature as $\eta_{i k}$. The (mean) light cone in state $\Psi$ can then be defined, for any space-time point, in terms of $g_{i k}(x \mid \Psi)$.

\section{Absolutely Space-Like Distances and Generalized Locality}

In classical gravitation theory, the causal structure of space-time is modified since Minkowski's metric $\eta_{i k}$ is replaced by the EinsteinRiemann metric $g_{i k}(x)$. Similar modifications have to be expected in a quantum theory of gravity.

The causal structure of Minkowski space-time leads to the usual locality postulate. Fields $\varphi_{\alpha}(x)$ and $\varphi_{\beta}(y)$ are required to commute (or anticommute) if the distance $y-x$ is space-like with respect to $\eta_{i k}$, i.e., with the signature used here, if

$$
\eta_{i k}\left(y^{i}-x^{i}\right)\left(y^{k}-x^{k}\right)<0 .
$$

In the presence of a quantized metric $g_{i k}(x)$, (5) does not necessarily forbid signal exchange between $x$ and $y$, since $g_{i k}(x)$ instead of $\eta_{i k}$ should determine the causal structure of space-time. Coordinate distances $y-x$ fulfilling (5) are therefore called formally space-like. A coordinate distance $y-x$ is called absolutely space-like, if signal exchange between $x$ and $y$ is forbidden even in the presence of the quantized metric $g_{i k}(x)$. Locality assumptions are justified only for absolutely space-like distances.

In the following heuristic discussion, expressions like $g_{i k}(x) a^{i} a^{k}$ with a fixed $c$-number vector $a$ are considered formally as observables 
at the point $x$. A measurement of $g_{i k}(x) a^{i} a^{k}$ in state $\Psi$ shall determine whether a neighboring point $x+d x$, with $d x$ parallel to $a$, can be causally connected with $x$ (case $g_{i k}(x) a^{i} a^{k} \geqq 0$ ) or not (case $g_{i k}(x) a^{i} a^{k}<0$ ). By definition, infinitesimal distances $d x$ are absolutely space-like if and only if they are parallel to vectors $a$ with $g_{i k}(x) a^{i} a^{k}$ negative definite (and without eigenvalue zero). $C$-number vectors $a$ with this property will therefore be called absolutely space-like directions. The crucial problem here is, of course, whether such directions may exist at all or, more generally, whether a field quantity like $g_{i k}(x) a^{i} a^{k}$ may be semibounded. This is as yet an open question.

Because of translation invariance, $g_{i k}(y) a^{i} a^{k}$ is unitarily equivalent to $g_{i k}(x) a^{i} a^{k}$, and is thus negative definite if and only if $g_{i k}(x) a^{i} a^{k}$ is. Therefore the absolutely space-like character of distances $d x$ and directions $a$ does not depend on the reference point $x$.

Finite absolutely space-like distances $y-x$ can not so easily be characterized in terms of $g_{i k}(x)$. Of course, they too are clearly translation invariant. Moreover, the following statement is obviously true:

Finite distances $y-x$ which have absolutely space-like direction, i.e., with $g_{i k}(x)\left(y^{i}-x^{i}\right)\left(y^{k}-x^{k}\right)$ negative definite, are absolutely space-like.

Indeed, if signal exchange is already forbidden for all infinitesimal distances $d x$ parallel to $y-x$, it must be also forbidden for the finite distance $y-x$ itself. The inverse is not conjectured to hold. By definition, a finite distance $y-x$ might well be absolutely space-like even if it does not have absolutely space-like direction, i.e., if signal exchange is possible for some sufficiently small fraction of the distance $y-x$.

The foregoing considerations should be made more rigorous by replacing the formal "observables" $g_{i k}(x) a^{i} a^{k}$ by $g_{i k}(f) a^{i} a^{k}$ with suitable non-negative test functions $f(x)$. More precisely, suitable selfadjoint extensions of $g_{i k}(f) a^{i} a^{k}$ have to be considered, provided they exist and are, in some physical sense, unique. Whereas the smearing with $f(x)$ is unproblematic in view of the translation invariance of absolutely space-like directions, the problem of self-adjoint extensions is as yet unsolved.

With the usual motivation, we will now generalize the locality postulate of axiomatic field theory [1] to the situation considered here. (The correct spin-statistics relation has now, in the absence of a proof, to be postulated.)

Postulate 4 (Generalized Locality). Smeared fields $\varphi_{\alpha}(f)$ and $\varphi_{\beta}(g)$ commute (or anticommute) on $D$, if $f(x) g(y) \neq 0$ for absolutely spacelike $y-x$ only. - 


\section{Quantum Fluctuations of the Light Cone}

The main result of this paper may be summarized as follows. Denote by $A$ (resp. $F^{\prime}$ ) the set of all absolutely (resp. formally) space-like directions. Then $A$ is a proper subset of $F$, i.e.,

$$
A \varsubsetneqq F .
$$

The relation $A \leqq F$ is obvious from Eq. (4). Let us therefore assume $A=F$. Then a contradiction can be derived by methods due to EPSTEIN, GLASER and JAFFE [10]. Since these authors consider the usual axiomatic framework which does not contain the theory investigated here, we will shortly repeat their argument, thereby convincing ourselves that the usual additional assumptions (temperedness and manifest Lorentz covariance of the fields) are not necessary for our purpose.

First of all, the well-known Reeh-Schlieder theorem remains valid here since our postulates are already sufficient for its proof [11]. According to this theorem, the algebra of field polynomials smeared with test functions which are zero outside some fixed open space-time region has the vacuum $\Psi_{0}$ as cyclic vector. Postulate 4 now implies the usual locality postulate, since by our hypothesis $A=F$ all formally spacelike distances must be absolutely space-like. From locality and ReehSchlieder theorem follows by standard arguments (compare [1]): The vacuum $\Psi_{0}$ is separating vector for fields $\varphi_{\alpha K}(x)$ or linear combinations $\varphi(x)$ thereof if they are smeared with test functions $f(x)$ with compact support, i.e., $\varphi(f) \Psi_{0}=0$ for such $f(x)$ implies $\varphi(f)=0$.

Consider a linear combination of fields $\varphi(x)$ with the following properties [10]:

i) $(\Psi, \varphi(f) \Psi) \geqq 0$ for all $\Psi \in D$ and some fixed test function $f(x)$ with compact support,

ii) $\left(\Psi_{0}, \varphi(f) \Psi_{0}\right)=0$.

These properties immediately imply [10] $\varphi(f) \Psi_{0}=0$, i.e., $\varphi(f)=0$. If i) and ii) hold for some set of test functions $f(x)$ whose linear combinations are dense in the whole test function space, they will imply $\varphi(g)=0$ for all test functions $g(x)$, i.e., $\varphi(x) \equiv 0$. Let us at this point make the additional assumption that the set of all non-negative test functions with compact support is already large enough to serve this purpose. (This assumption is satisfied, of course, for the test function spaces $\mathscr{D}$ and $\mathscr{S}$.)

Choose now an arbitrary formally light-like direction $a$, i.e., $\eta_{i k} a^{i} a^{k}=0$, and consider $\varphi(f)=-g_{i k}(f) a^{i} a^{k}$ with an arbitrary nonnegative test function $f(x)$ with compact support. By $A=F$, a formally space-like direction $b$ is also absolutely space-like, and is thus space-like with respect to $g_{i k}(x \mid \Psi)$ for any state $\Psi \in D$. The formally light-like 
direction $a$ is then, with respect to $g_{i k}(x \mid \Psi)$, space-like or at most lightlike. This implies property i) above. Property ii) follows from Eq. (4). Therefore $g_{i k}(f) a^{i} a^{k}=0$ for all non-negative $f(x)$ with compact support, and thus $g_{i k}(x) a^{i} a^{k}=0$ for an arbitrary formally light-like direction $a$, i.e., $g_{i k}(x)$ is conformally flat. Such $g_{i k}(x)$, however, can not describe a quantized field of gravity. This is the desired contradiction.

According to relation (6), the local light cone $g_{i k}(x) d x^{i} d x^{k}=0$ of the quantized metric $g_{i k}(x)$ exhibits quantum fluctuations which necessarily extend to certain formally space-like directions. In other words, the light cone at $x$ of $g_{i k}(x \mid \Psi)$ must include, for some states $\Psi \in D$, certain points which are formally space-like with respect to $x$. Therefore generalized locality (Postulate 4) is, at least for sufficiently small distances $y-x$, a weaker assumption than the usual locality postulate. It may be even vacuous, if there are no absolutely space-like distances at all. (Note, however, that the non-existence of absolutely space-like directions does not necessarily imply the non-existence of absolutely space-like distances.)

The linear static approximation of the classical Einstein theory yields, in harmonic coordinates, the local velocity of light

$$
c^{\prime}=c\left(1+2 V / c^{2}\right)
$$

with Newton's gravitational potential $V$ (see, e.g., [5], Ch. 5, § 55). For non-negative mass densities, $V \leqq 0$ and therefore $c^{\prime} \leqq c$, i.e., formally space-like directions remain space-like also in the gravitational field. The relation (6) excludes such possibility for quantum theories of gravitation which satisfy our general assumptions. However, this is not too surprising in view of the approximate nature of the classical result (7) and the non-existence of positive mass densities in quantum field theory [10].

Moreover, harmonic coordinates define a covariant gauge, and the quantized version of the theory [7] therefore does not belong to the class of theories considered here. In covariant gauges, manifest Lorentz covariance of $g_{i k}(x)$ implies Lorentz invariance of the set $A$ of absolutely space-like directions. By this, together with Eq. (4), either $A=F$, or $A$ is empty. Since our result (6) is not likely to apply to this case, we can not yet exclude the possibility $A=F$ for such theories.

Acknowledgements. I want to thank Profs. G. LUdwig, K. JUST and D. R. BRILL for exciting and encouraging discussions, and Prof. R. HAAG and Dr. G. C. HeGERFELDT for useful comments on a preliminary version of this paper. Financial support from the Deutsche Forschungsgemeinschaft is also gratefully acknowledged. 


\section{References}

1. Streater, R. F., and A. S. Wightman: PCT, spin and statistics, and all that. New York-Amsterdam: W. A. Benjamin 1964.

2. Strocchi, F.: Phys. Rev. 166, 1302 (1968).

3. JAFFE, A.: Phys. Rev. 158, 1454 (1967).

4. Wightman, A. S.: Phys. Rev. 101, 860 (1956).

5. Fock, V.: The theory of space time and gravitation. London-New YorkParis-Los Angeles: Pergamon Press 1959.

6. Arnowitt, R. L., S. Deser, and C. W. Misner in: Recent developments in general relativity, p. 127. New York: Pergamon Press 1962, and further work quoted there.

7. Gupta, S. N.: Proc. Phys. Soc. A 65, 161, 608 (1952).

Just, K.: Nuovo Cimento 34, 567 (1964).

8. Strocchr, F.: Phys. Rev. 162, 1429 (1967).

9. Weinberg, S.: Brandeis lectures 1964, Vol. II, p. 405, and further work quoted there.

10. Epstein, H., V. Glaser, and A. JAFFE: Nuovo Cimento 36, 1016 (1965).

11. REEH, H., and S. Schlieder: Nuovo Cimento 22, 1051 (1961).

K. KraUs,

Institut für Theoretische Physik (I)

der Universität

3550 Marburg, Renthof 7 